\title{
The Regulation of Mesangial Cell Proliferation
}

\author{
Andrea Cove-Smith Bruce M. Hendry \\ Renal Medicine, King's College London, Weston Education Centre, London, UK
}

\section{Key Words}

Cell cycle $\cdot$ Renal $\cdot$ Glomerular $\cdot$ Nephritis $\cdot$ Ras $\cdot$ Calcium

\begin{abstract}
Mesangial cell (MC) proliferation is a key pathological feature in a number of common human renal diseases including IgA, systemic lupus erythematosus and diabetic nephropathies. Knowledge of the role of MCs in normal glomeruli and of their response to pathological stimuli is crucial to the understanding of these disease processes. The purpose of understanding disease is ultimately to develop therapeutic strategies that can limit or even reverse the underlying pathological process. Over the last 20 years a number of signaling pathways involved in the regulation of MC proliferation have been identified and studied with a view to manipulating them for therapeutic gain. Unfortunately, despite these advances, there are still very few clinical options that specifically target aberrant MC proliferation. This article reviews a number of factors that have been shown to play a role in controlling $\mathrm{MC}$ proliferation, including signaling molecules (e.g. Platelet-derived growth factor, Ras and $\mathrm{Ca}^{2+}$ ), cell cycle proteins (e.g. cyclin D1) and transcription factors (E2F). A variety of strategies has been used to manipulate these different pathways to elucidate their function in MCs with the ultimate aim of modifying them in order to treat human renal diseases.

Copyright $\odot 2008$ S. Karger AG, Basel
\end{abstract}

\section{Introduction}

Aberrant proliferation of mesangial cells (MCs) is a common finding in a number of diseases that can lead to end-stage renal failure. A variety of initial insults, which may be metabolic (as in diabetic nephropathy), or immunological (as in IgA disease and lupus nephritis), can cause uncontrolled MC proliferation. This in turn causes an increase in extracellular matrix (ECM) deposition and ultimately leads to glomerulosclerosis, with subsequent reduction in glomerular filtration rate due to loss of functioning nephrons. It is important to understand the role of MCs in the healthy kidney in order to determine the key regulatory mechanisms involved in this pathological response to injury and ultimately to identify potential targets for therapeutic intervention.

Understanding the role of MCs, both in healthy kidney and in disease states, is fraught with difficulties that are common to the study of any complex biological system. Evidence of the various functions of MCs, and the factors involved in controlling these functions, may come from a number of sources, including in vitro studies using primary cell cultures or cell lines, ex vivo studies using isolated glomeruli, or in vivo disease models. The difficulty lies in the appropriate interpretation of these data; just because MCs can behave in a certain manner in response to a particular agent in vitro does not necessarily

\section{KARGER \\ Fax +4161306 1234 E-Mail karger@karger.ch} www.karger.com
(C) 2008 S. Karger AG, Basel $1660-2129 / 08 / 1084-0074 \$ 24.50 / 0$

Accessible online at: www.karger.com/nee
Bruce M. Hendry

Renal Medicine, King's College London

Weston Education Centre

London SE5 9PJ (UK)

Tel. +44 207848 0439, Fax +44 207848 0515, E-Mail bruce.hendry@kcl.ac.uk 
mean that they do respond this way in vivo, or that this is a biologically significant interaction. Moreover, it is well recognized that cell lines may respond differently to certain stimuli than would primary cultures of the same cell type. This is particularly pertinent to the study of proliferation, the control of which is, by definition, altered in the generation of stable cell lines. It is also crucial that the appropriate model is used to study particular pathways. For example, it has been shown that the role of specific Ras isoforms in the regulation of proliferation and apoptosis differs between mouse and human MCs [1]. This suggests that mouse models of mesangial proliferative disease may not be appropriate surrogates for their human equivalent, at least in this context. With these caveats in mind this paper aims to review the evidence supporting the better understood roles of MCs and the involvement of some of the more extensively studied signaling molecules, as well as some novel pathways, in these processes. The focus is on those signaling pathways that have been shown to be relevant in vivo and in vitro, ideally in both human and animal MCs, to provide a degree of confidence that they are of some biological significance in the control of MC proliferation.

\section{Role of Mesangial Cells}

\section{Structural Support and Secretion of ECM}

The most well established role of MCs is that of providing structural support to the glomerular tuft, in part by the secretion and maintenance of the extracellular matrix. In the healthy glomerulus, components of the ECM are synthesized and degraded in a balanced manner, ensuring that the quantity and quality of the ECM is maintained throughout adult life. The production of ECM by MCs is controlled primarily by cytokines such as interleukin-1 (IL-1), platelet-derived growth factor (PDGF) and transforming growth factor beta (TGF- $\beta$ ) [2].

This structural role of the mesangium is clearly very important, but the glomerular ECM is thought to be more than simply a passive structural scaffold. In vitro studies have shown that the components of the ECM have the ability to directly influence the behavior of the MCs with which they are in contact. For example, freshly seeded MCs have a significantly increased replication rate when plated on collagens I, II and IV, and cells cultured on laminin and collagen IV show an increase in fibronectin and laminin secretion [2]. MCs are known to express integrins on their surface and therefore have the capacity to bind certain components of the ECM and respond di- rectly to them. It is also possible that the ECM may sequester soluble cytokines and present them to adjacent cells in a more biologically active form. Thus it seems that the ECM is not only secreted by glomerular MCs, but also has the potential ability to influence their behavior with respect to proliferation, migration and ECM production.

\section{Modulation of Glomerular Filtration}

MCs have the characteristics of modified smooth muscle cells and have been thought to have a role in modulating glomerular filtration since the early anatomical studies in the 1920s. More recently, it has been postulated that this function is made possible by the response of MCs to various vasoactive stimuli [3]. These stimuli may be circulating substances such as angiotensin II or locally generated agents such as prostaglandins which have been shown to cause contraction of MCs in vitro. The contraction of MCs surrounding capillary loops will cause them to constrict, altering the blood flow to certain areas within the glomerular tuft and thus influencing intraglomerular hemodynamics.

\section{Phagocytosis of Macromolecules and Immune Complexes}

Electron microscopic studies by Farquhar and Palade in the 1960s demonstrated that MCs are capable of taking up macromolecules. Further experiments by Schlondorff et al. in the 1980s showed that this process involves a typical coated pit/coated vesicle mechanism of endocytosis with delivery of the particles ultimately to phagolysosomes [3]. This results in the generation of inflammatory mediators such as prostaglandins and oxygen radicals. Resident macrophages also play a major role in the uptake of macromolecules and immune complexes, and the interaction between these two cell types is an important component in controlling glomerular inflammation.

\section{Response to and Secretion of Cytokines}

There is very little MC turnover in the healthy adult kidney, suggesting that under normal circumstances MCs are either not exposed to mitogens or are protected from them by inhibitory factors. However, in vitro, MCs are capable of both producing and responding to a number of cytokines including IL-1 $\beta$, tumor necrosis factor alpha (TNF- $\alpha$ ), PDGF and basic fibroblast growth factor (bFGF). These substances can act in a paracrine fashion as they are synthesized and secreted by activated macrophages, or in an autocrine manner as they can also be secreted by MCs themselves. After glomerular injury, 
Fig. 1. Key pathways in the control of MC proliferation and some opportunities for intervention to reduce proliferation. See text for details.

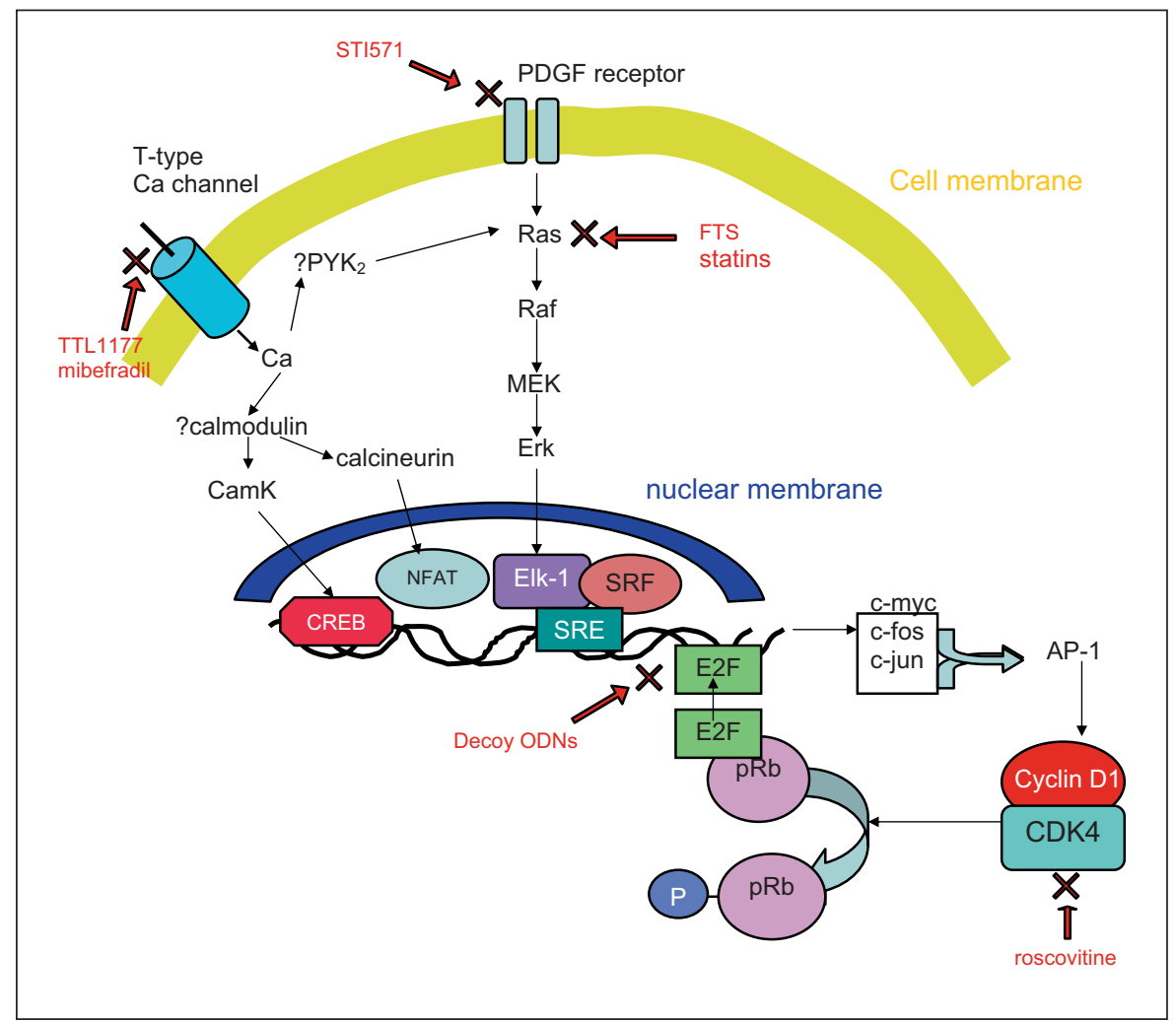

these cytokines may initially serve to stimulate appropriate repopulation of the glomerular tuft with MCs, but in disease they seem to sustain the inflammatory process, thereby promoting ongoing MC proliferation and ECM production leading to progressive glomerular damage.

\section{Regulation of Mesangial Cell Proliferation in Disease}

\section{PDGF}

PDGF is a potent mitogen for cells of mesenchymal origin. In 1988, Abboud et al. demonstrated that human and rat MCs are capable of synthesizing and releasing PDGF and also that binding of PDGF to human MCs stimulates proliferation [4]. Since that time PDGF has been widely studied in both human and experimental mesangial proliferative renal disease.

In vivo, infusion of PDGF or bFGF induces MC proliferation and matrix accumulation in rat kidneys. Moreover, PDGF expression in glomeruli increases in parallel with MC proliferation in the well-studied Thyl nephritis model in rats. In this model, a single injection of antiThyl monoclonal antibody leads to an acute complementdependent loss of MCs with disruption of the mesangial matrix. Subsequently, there is a marked increase in MC proliferation (from day 2-3 post antibody injection), along with extracellular matrix accumulation and infiltration of monocytes into the glomerular tuft. Administering neutralizing anti-PDGF IgG prior to disease induction results in a significant reduction in MC proliferation and ECM deposition in this model [5]. Inhibition of PDGF using STI 571, a selective PDGF receptor tyrosine kinase inhibitor, has been shown to inhibit MC proliferation in this disease as well as in cultured MCs in vitro.

PDGF binds to a tyrosine kinase receptor which activates a number of downstream effectors, including the Ras/MAPK pathway, to activate various transcription factors and proto-oncogenes associated with cell proliferation. These transcription factors include c-fos, c-myc and c-jun. C-fos dimerizes with c-jun to form the AP-1 complex which transactivates a number of target genes including Cyclin D1 (fig. 1). Cyclin D1, along with its associated cyclin-dependent kinases (CDK4 and 6), is a key regulatory protein in controlling the reentry of quiescent cells in G0 into the cell cycle at G1. The cyclin-CDK com-

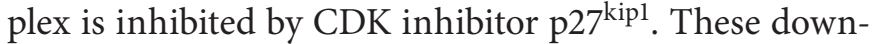
stream effectors have increasingly been studied and a number of strategies have been used to manipulate them 
in order to modify MC proliferation both in vitro and in vivo.

\section{Cyclins, CDKs and CDK Inhibitors}

The Thyl nephritis model has again been invaluable in studying the changes in cell cycle proteins during experimentally induced MC proliferation in vivo. In this model, the levels of cyclin D1, CDK4 and CDK2 all increase during the phase of marked mesangial proliferation. The role of cyclin D1 has been investigated further in MCs in vitro by the use of antisense oligonucleotides (ODNs). Reduction of the serum or PDGF-stimulated expression of cyclin D1 using ODNs leads to a marked reduction in MC proliferation as assessed by $\left[{ }^{3} \mathrm{H}\right]$-thymidine uptake. This effect correlates with diminished CDK4 activity [6].

The biological relevance of increased CDK levels in Thyl nephritis has been studied using roscovitine, a purine analogue which competitively inhibits kinase activity by binding to the ATP-binding site of CDKs. Administration of roscovitine either immediately after disease induction or once mesangial proliferation is fully established, significantly reduces MC proliferation. This is associated with a reduction in ECM protein expression and an improvement in renal function [7].

In normal rat glomeruli the expression of the CKI $\mathrm{p} 27^{\mathrm{kip} 1}$ is high, in keeping with the quiescent nature of MCs in healthy kidney. At the onset of MC proliferation in the Thy1 model, the levels of p27 ${ }^{\mathrm{kipl}}$ decrease dramatically. If experimental nephritis is induced in p27 $7^{\mathrm{kip}}{ }_{-} /-$ mice, the onset of mesangial proliferation occurs earlier and the magnitude of the proliferative response, coupled with ECM expansion, is greater. Again, this correlates well with in vitro findings that quiescent MCs express p2 $7^{\mathrm{kip} 1}$ and that this expression is reduced upon PDGF and bFGF-induced MC proliferation. Moreover, reducing the levels of p27 $7^{\mathrm{kip} 1}$ using ODNs in vitro results in an increased magnitude of MC proliferation in response to mitogens, although $\mathrm{p} 27^{\mathrm{kip} 1}$ reduction alone has no effect [8].

The conjunction of cyclin D1 with CDK4 and cyclin E with CDK2 facilitates phosphorylation of retinoblastoma protein $(\mathrm{pRb})$, releasing E2F transcription factors. This family of transcription factors regulates cell cycle progression by upregulating the expression of proteins required for the G1/S phase transition and DNA synthesis, such as cyclin $\mathrm{A}$, cyclin $\mathrm{E}$, proliferating cell nuclear antigen (PCNA) and c-myc. Decoy ODNs containing the consensus E2F-binding sequence have been transfected into MCs to act as competitive inhibitors of this tran- scription factor. In vitro, transfection of these decoy ODNs effectively reduces serum-stimulated MC proliferation. In vivo, administration of E2F ODNs via the left renal artery $36 \mathrm{~h}$ after the induction of Thyl nephritis causes a reduction in glomerular cellularity compared to mismatch control ODNs. This effect was associated with a reduction in the expression of PCNA at both mRNA and protein level [9].

\section{Ras}

The Ras family of monomeric GTPases are intracellular signaling molecules which act as molecular switches downstream from tyrosine kinase receptors including the PDGF receptor. Work in our lab has shown that PDGFinduced proliferation of human MCs in vitro requires both Ki-Ras and Ha-Ras. S-trans, trans-farnesylthiosalicylic acid (FTS) is a synthetic S-prenyl derivative of a rigid carboxylic acid. This resembles the carboxy-terminal farnesylcysteine group common to all Ras proteins and therefore acts as a functional Ras antagonist by affecting interactions of Ras with the cell membrane. Dislodging Ras from its anchorage domains results in increased degradation thereby reducing cellular Ras content and downregulating Ras-dependent signaling functions.

In vitro, FTS reduces PDGF-stimulated rat and human MC proliferation [10], and in vivo, glomerular expression of both Ki-Ras and N-Ras increases in Thy-1 nephritis. Moreover, the use of FTS to treat Thy-1 nephritis results in a significant reduction in actively proliferating glomerular cells as assessed by BrdU uptake, along with a reduction in glomerular macrophage number and proteinuria 10 days after disease induction [11].

Simvastatin has been shown to inhibit MC proliferation both in vitro and in vivo [12]. Statins act by inhibiting 3-hydro-3-methylglutaryl coenzyme A (HMG-CoA) reductase and were initially developed as lipid-lowering agents. However, some of their beneficial effects appear to be independent of cholesterol reduction. Inhibition of HMG-CoA reductase causes a reduction in intermediate metabolites of the mevalonate pathway such as farnesyl pyrophosphate (FPP) and geranylgeranyl pyrophosphate (GGPP). Both of these metabolites are important anchors for both the Ras and Rho families, suggesting that some of the impact of statins on the cell cycle may be via downregulation of the Ras/Rho-dependent signaling pathways.

\section{Calcium-Dependent Mechanisms}

T-type calcium channels are low-voltage activated, transient current calcium channels that have recently 
been shown to have a crucial role in controlling cell cycle progression in prostate cancer cells and vascular smooth muscle cells [13]. Three isoforms exist which differ slightly in the structure of their pore-forming alpha subunits and are known as $\alpha_{1} G, \alpha_{1} H$ and $\alpha_{1}$ I. Studies in our lab have shown that human MCs express mRNA for the $\alpha_{1} \mathrm{H}$ isoform and also that these cells have recordable T-type calcium currents. Furthermore, inhibition of these channels either pharmacologically (using mibefradil, TTL1177 or Nickel chloride) or by RNA interference is antiproliferative in human cells in vitro. In vivo, induction of Thy1 nephritis in rats causes an increase in mRNA expression for the $\alpha_{1} H$ isoform (unpublished data C.J. Mulgrew, B.M. Hendry).

Little is understood about how these channels interact with the cell signaling pathways to regulate cell cycle progression. One unresolved issue is how the small inward flux of calcium through these channels is distinguished by the cell from other, larger intracellular calcium transients. It may be that the necessary signaling machinery is in some way spatially associated with the T-type current and therefore ideally placed to detect these changes, or that the temporal pattern of $\mathrm{Ca}^{2+}$ transients determines the specificity of the cellular response. What has been shown, by our group and others, is that pharmacological inhibition of T-type channels or knockdown of the $\alpha_{1} \mathrm{H}$ isoform causes MCs to accumulate in G1 with a reduced proportion of cells in S-phase. This suggests a role for T-type calcium channels in controlling progression through the G1/S boundary. Two signaling pathways that have been implicated in this are the Ras/MAPK cascade via activation of a proline-rich tyrosine kinase $\left(\mathrm{PYK}_{2}\right)$, or calmodulin which can activate calmodulindependent kinases (e.g. CaMKII) and calcineurin.

Calmodulin $(\mathrm{CaM})$ is a highly conserved $\mathrm{Ca}^{2+}$-binding protein which regulates a number of functions in mammalian cells. Its expression increases as cells approach the G1/S boundary of the cell cycle and over expression of CaM causes accelerated cell proliferation due to a shortening of G1 [14]. $\mathrm{Ca}^{2+} / \mathrm{CaM}$ activates both CaMKII and calcineurin via the relief of autoinhibition. Once activated, both of these proteins are known to have a role in activating transcription factors $\left(\mathrm{Ca}^{2+} / \mathrm{cAMP}\right.$ response element binding protein (CREB) and NFAT, respectively) that are important in cell cycle progression. CaMKII in turn is able to phosphorylate T-type channels themselves, causing increased opening frequency and therefore potentially driving a feedforward mechanism [15]. These candidate pathways require further study in MCs to determine their role in T-type $\mathrm{Ca}^{2+}$ channel signaling.

\section{Future Therapies}

As the mechanisms involved in regulating MC proliferation are increasingly understood, the aim is to identify potential targets that can be manipulated to produce therapeutic benefit. A number of hurdles remain to be overcome in order to achieve this goal. It can be assumed that a degree of MC proliferation must be beneficial in certain circumstances, for example in allowing resolution of transient glomerulopathies such as post-streptococcal GN in human disease or indeed experimental Thy1 nephritis in rats which, if left to run its course, resolves within a few weeks. Any therapy designed to inhibit MC proliferation may potentially limit these beneficial healing processes. Therefore, like many strategies in medicine, the ultimate goal is to strike a balance whereby the appropriate reconstitutive proliferation of MCs can still occur, but the pathological, uncontrolled proliferation and accompanying increase in ECM is minimized.

Targeting ubiquitous intracellular signaling molecules such as Ras or using non-specific kinase inhibitors such as roscovitine have shown promise in animal models but may result in off-target effects and therefore potentially lead to significant toxicity. It would be desirable to inhibit mechanisms that are unique to proliferating MCs to avoid effects on other actively dividing cells, but as most important signaling mechanisms are utilized in numerous cell types, this may prove an unrealistic aim. It is also a concern that, given the redundancy present in many biological systems, targeting one specific molecule or pathway may result in compensatory upregulation of other mechanisms.

However, it is encouraging to note that many investigators have been able to inhibit MC proliferation both in vitro and in vivo, using a number of different strategies. It is particularly promising that some of these strategies have been shown to improve renal function and reduce proteinuria in animal models and have not resulted in significant toxicity. Simvastatin is in regular clinical use, as is benidipine (a combined T- and L-type $\mathrm{Ca}^{2}$ channel blocker), and both agents may have additional benefit on limiting MC proliferation, over and above their well-documented effects on lipid metabolism and hypertension, respectively. Furthermore, FTS and new generations of CDK-inhibiting drugs are being increasingly studied and modified as potential therapies for cancer, but may also provide promising new strategies in the treatment of mesangial proliferative renal disease. 


\section{References}

1 Hendry BM, Khwaja A, et al: Distinct functions for Ras GTPases in the control of proliferation and apoptosis in mouse and human mesangial cells. Kidney Int 2006;69: 99-104.

2 Sterzel RB, Schulze-Lohoff E, et al: Interactions between glomerular mesangial cells, cytokines, and extracellular matrix. J Am Soc Nephrol 1992;2(10 suppl):S126-S131.

$\checkmark 3$ Schlondorff D: The glomerular mesangial cell: an expanding role for a specialized pericyte. Faseb J 1987;1:272-281.

4 Shultz PJ, DiCorleto PE, et al: Mesangial cells express PDGF mRNAs and proliferate in response to PDGF. Am J Physiol 1988;255: F674-F684.

5 Johnson RJ, Raines EW, et al: Inhibition of mesangial cell proliferation and matrix expansion in glomerulonephritis in the rat by antibody to platelet-derived growth factor. J Exp Med 1992;175:1413-1416.
6 Schocklmann HO, Lang S, et al: Regulation of mesangial cell proliferation. Kidney Int 1999;56:1199-1207.

7 Pippin JW, Qu Q, et al: Direct in vivo inhibition of the nuclear cell cycle cascade in experimental mesangial proliferative glomerulonephritis with Roscovitine, a novel cyclin-dependent kinase antagonist. J Clin Invest 1997;100:2512-2520.

8 Marshall CB, Shankland SJ: Cell cycle and glomerular disease: a minireview. Nephron Exp Nephrol 2006;102:e39-e48.

$\checkmark 9$ Maeshima Y, Kashihara N, et al: Inhibition of mesangial cell proliferation by E2F decoy oligodeoxynucleotide in vitro and in vivo. J Clin Invest 1998;101:2589-2597.

10 Khwaja A, Sharpe CC, et al: The inhibition of human mesangial cell proliferation by Strans, trans-farnesylthiosalicylic acid. Kidney Int 2005;68:474-486.

-11 Clarke HC, Kocher HM, et al: Ras antagonist farnesylthiosalicylic acid (FTS) reduces glomerular cellular proliferation and macrophage number in rat thy-1 nephritis. J Am Soc Nephrol 2003;14:848-854.
12 Yoshimura A, Inui K, et al: Simvastatin suppresses glomerular cell proliferation and macrophage infiltration in rats with mesangial proliferative nephritis. J Am Soc Nephrol 1998;9:2027-2039.

13 Rodman DM, Reese K, et al: Low-voltage-activated (T-type) calcium channels control proliferation of human pulmonary artery myocytes. Circ Res 2005;96:864-872.

14 Kahl CR, Means AR: Regulation of cell cycle progression by calcium/calmodulin-dependent pathways. Endocr Rev 2003;24:719736 .

15 Yao J, Davies LA, et al: Molecular basis for the modulation of native T-type $\mathrm{Ca} 2+$ channels in vivo by $\mathrm{Ca} 2+/$ calmodulin-dependent protein kinase II. J Clin Invest 2006;116: 2403-2412.

\section{Erratum}

In the article by D. Cibulskyte et al. entitled 'Renal Effects of Long-Term Ciclosporin A Treatment in a Large Animal Model' [Nephron Exp Nephrol 2007;105:e91-e97] the correct citation of the author Ringer Ellingsen A. should be Ellingsen A.R. 